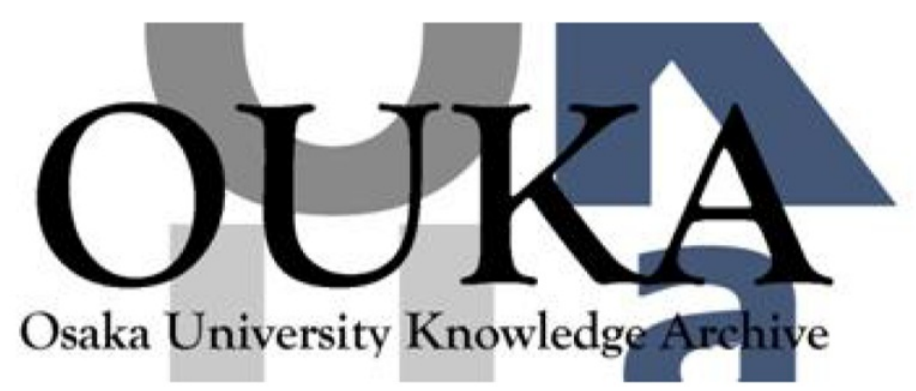

\begin{tabular}{|c|c|}
\hline Title & $\begin{array}{l}\text { Effect of Relativistic Plasma on Extreme- } \\
\text { Ultraviolet Harmonic Emission from Intense } \\
\text { Laser-Matter Interactions }\end{array}$ \\
\hline Author (s) & Krushelnick, K.; Rozmus, W.; Wagner, U. et al. \\
\hline Citation & $\begin{array}{l}\text { Physical Review Letters. 100(12) p. 125005-1- } \\
\text { p. 125005-4 }\end{array}$ \\
\hline Issue Date & $2008-03-28$ \\
\hline oaire:version & VoR \\
\hline URL & https://hdl. handle. net/11094/3425 \\
\hline rights & $\begin{array}{l}\text { Krushelnick, K., Rozmus, W., Wagner, U., Beg, } \\
\text { F. N., Bochkarev, S. G., Clark, E. L., Dangor, } \\
\text { A. E., Evans, R. G., Gopal, A., Habara, H., } \\
\text { Mangles, S. P. D., Norreys, P. A. , Robinson, A. } \\
\text { P. L., Tatarakis, M., Wei, M. S.,' Zepf, M. ' } \\
\text { Physical Review Letters, 100, 12, 125005, 2008- } \\
\text { 03-28. "Copyright } 2008 \text { by the American Physical } \\
\text { Society." }\end{array}$ \\
\hline Note & \\
\hline
\end{tabular}

Osaka University Knowledge Archive : OUKA

https://ir. Library. osaka-u. ac. jp/

0saka University 


\title{
Effect of Relativistic Plasma on Extreme-Ultraviolet Harmonic Emission from Intense Laser-Matter Interactions
}

\author{
K. Krushelnick, ${ }^{1, *}$ W. Rozmus, ${ }^{2}$ U. Wagner, ${ }^{3}$ F. N. Beg, ${ }^{4}$ S. G. Bochkarev, ${ }^{2,5}$ E. L. Clark, ${ }^{6}$ A. E. Dangor, ${ }^{1}$ R. G. Evans, ${ }^{1,3}$ \\ A. Gopal,${ }^{6}$ H. Habara, ${ }^{3}$ S. P. D. Mangles, ${ }^{1}$ P. A. Norreys, ${ }^{3}$ A. P. L. Robinson,${ }^{1,3}$ M. Tatarakis, ${ }^{1,6}$ M. S. Wei, ${ }^{4}$ and M. Zepf ${ }^{7}$ \\ ${ }^{1}$ The Blackett Laboratory, Imperial College, London SW7 2AZ, United Kingdom \\ ${ }^{2}$ Department of Physics, University of Alberta, Edmonton Canada \\ ${ }^{3}$ Rutherford Appleton Laboratory, Chilton, Oxon, OX11 0QX, United Kingdom \\ ${ }^{4}$ Department of Mechanical and Aerospace Engineering, University of California, San Diego, California 92093-0411, USA \\ ${ }^{5}$ Lebedev Physical Institute, Russian Academy of Sciences, Moscow, Russia \\ ${ }^{6}$ Technological Educational Institute, Chania, Crete, Greece \\ ${ }^{7}$ Department of Physics, The Queens University, Belfast BT7 1NN, United Kingdom
}

(Received 11 August 2006; published 28 March 2008)

\begin{abstract}
Experiments were performed in which intense laser pulses (up to $9 \times 10^{19} \mathrm{~W} / \mathrm{cm}^{2}$ ) were used to irradiate very thin (submicron) mass-limited aluminum foil targets. Such interactions generated high-order harmonic radiation (greater than the 25th order) which was detected at the rear of the target and which was significantly broadened, modulated, and depolarized because of passage through the dense relativistic plasma. The spectral modifications are shown to be due to the laser absorption into hot electrons and the subsequent sharply increasing relativistic electron component within the dense plasma.
\end{abstract}

DOI: 10.1103/PhysRevLett.100.125005

Recent advances in laser technology have enabled the development of petawatt-class laser facilities capable of intensities greater than $10^{20} \mathrm{~W} \mathrm{~cm}^{-2}$. Such experiments have led to observations of beams of relativistic electrons [1], $\gamma$ rays [2], and energetic protons [3] as well as laser induced nuclear reactions [4] and huge magnetic fields [5]. However, because of the small spatial and temporal scales of the dense plasmas produced it remains difficult to diagnose these interactions precisely using conventional techniques.

During such experiments, measurements of harmonic emission of the fundamental laser frequency can be a powerful diagnostic of the plasma conditions in the interaction [5-8]. High-order harmonics are useful since they are generated from the same time and region as the intense laser interaction. The generation mechanism for these harmonics is also well understood $[9,10]$, so it is possible to infer a significant amount of information by such measurements.

In this Letter we discuss an experiment at ultrahigh intensities in which the detailed spectrum and the polarization properties of high-order harmonics were measured after propagation through high density relativistic plasma. The spectrum of harmonic emission observed at the rear of initially very thin targets exhibits significant broadening, redshifting, and multiple peak structure when compared to harmonics measured at the front of the plasma. We also observe a difference in the spectrum of separate polarization components. The measured spectral broadening and frequency shifting of the harmonics is shown here to be likely due to the temporal variations in the index of refraction within the plasma which are caused by the rapidly changing composition of the target plasma such that relativistic electrons become the dominant component during
PACS numbers: $52.70 . \mathrm{Kz}, 52.27 . \mathrm{Ny}, 52.38 .-\mathrm{r}, 52.50 . \mathrm{Jm}$

the time period of the laser pulse as a result of high conversion of laser energy into these electrons $[1,11]$. Such observations may lead to a method for in situ measurements of the properties of dense relativistic plasmas which are of critical importance for the evaluation of fast ignition for inertial confinement fusion [12].

These experiments were performed using the Vulcan 100 TW laser system at the Rutherford Appleton Laboratory, which produces $p$-polarized $0.7-1.0$ ps pulses at $1.053 \mu \mathrm{m}$ with energies up to $70 \mathrm{~J}$. The $20 \times 11 \mathrm{~cm}^{2}$ beam was focused by an off axis parabolic mirror $(f=$ $300 \mathrm{~mm}$ ). The targets for this experiment consisted of very thin aluminum $(0.2$ and $0.4 \mu \mathrm{m})$. The peak intensity was up to $I_{0} \sim 9 \times 10^{19} \mathrm{~W} \mathrm{~cm}^{-2}$ and the laser prepulse (nanoseconds before the main pulse) was determined to be $10^{-6}$ of the peak intensity using third order autocorrelation techniques (the prepulse $\sim 50 \mathrm{ps}$ before the main pulse had a contrast of about $10^{-4}$ ) [13]. The subcritical plasma produced by this prepulse in front of the target was measured by optical probing to have a scale length less than $5 \mu \mathrm{m}[1]$.

The plasma density profile of the preheated foil was simulated using the two-dimensional hydrocode POLLUX [14] using 0.2 and $0.4 \mu \mathrm{m}$ thick aluminum foils which were preheated by the prepulse which determined the plasma conditions at the time of the interaction with the main pulse. In the $0.2 \mu \mathrm{m}$ case the plasma expands to a thickness of about $5 \mu \mathrm{m}$ and the density peaked at about 10 times the critical density (i.e., $\sim 10^{22} \mathrm{~cm}^{-3}$ ) while in the case of the $0.4 \mu \mathrm{m}$ thick foils the peak plasma density was about $7 \times 10^{22} \mathrm{~cm}^{-3}$. The thickness of the dense region was $10 \mu \mathrm{m}$ in this case. The pulse "shoulder" had a smaller effect on the target plasma conditions. It should be noted that the plasma conditions at the front of the target 


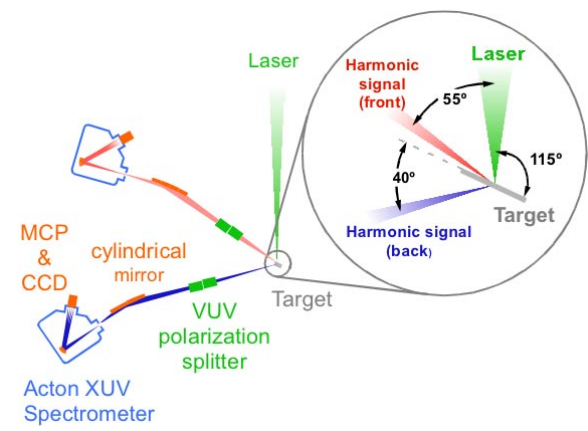

FIG. 1 (color online). Experimental setup.

(up to the critical density) are similar for $0.2 \mu \mathrm{m}$ thick foils, $0.4 \mu \mathrm{m}$ thick foils, and slab targets - indicating that for different thickness targets the effect of the prepulse is primarily to change the peak density of the target plasma and the thickness of the target plasma.

In these experiments, two multichannel vacuum ultraviolet (vuv) polarimeters were constructed to perform simultaneous spectral and polarization measurements of harmonic emission (order $>6$ ). The vuv polarizers use a triple-mirror configuration which polarizes light due to the Fresnel reflections on gold-coated mirror surfaces. Two vuv polarizers were set up orthogonally to measure the $p$ and the $s$ components of the harmonics. The polarized beams are then imaged onto the slit of a vuv spectrometer, enabling the simultaneous measurement of the different polarizations over many harmonic orders. A microchannel plate coupled to a charge-coupled device by a fiber-optic bundle was used as the detector. One vuv polarimeter was positioned at the rear of the aluminum foil target at an angle of $50^{\circ}$ to the target normal and the harmonic spectrum was measured during interactions at peak laser intensity. A second was positioned to measure the harmonics simultaneously from the front surface (see Fig. 1).

Examples of data acquired during the experiment are shown in Fig. 2. Figure 2(a) shows an example of a spectrum taken from the front of the target while Fig. 2(b) shows the spectrum of the harmonics from the rear of the interaction with a $0.2 \mu \mathrm{m}$ thick $\mathrm{Al}$ target. Figure 2(c) shows the rear spectrum using a thicker target plasma $(0.4 \mu \mathrm{m} \mathrm{Al})$.

The principal observation from this experiment is the significant reproducible broadening and redshifting of the harmonics observed after propagation through the plasma which is dependent on harmonic order. Also of particular interest is the modulation observable on the harmonics in Fig. 2(b) which was observed to be highly polarization dependent. For thicker targets [Fig. 2(c)] the broadening of the harmonics almost washes out all of the spectral structure.

Earlier experiments [15] at much lower power have shown that transmitted harmonics can be used as a density diagnostic for the thin region of dense plasma behind the main interaction. And more recent experiments at lower power have also shown that for intensities $\sim 10^{18} \mathrm{~W} / \mathrm{cm}^{2}$
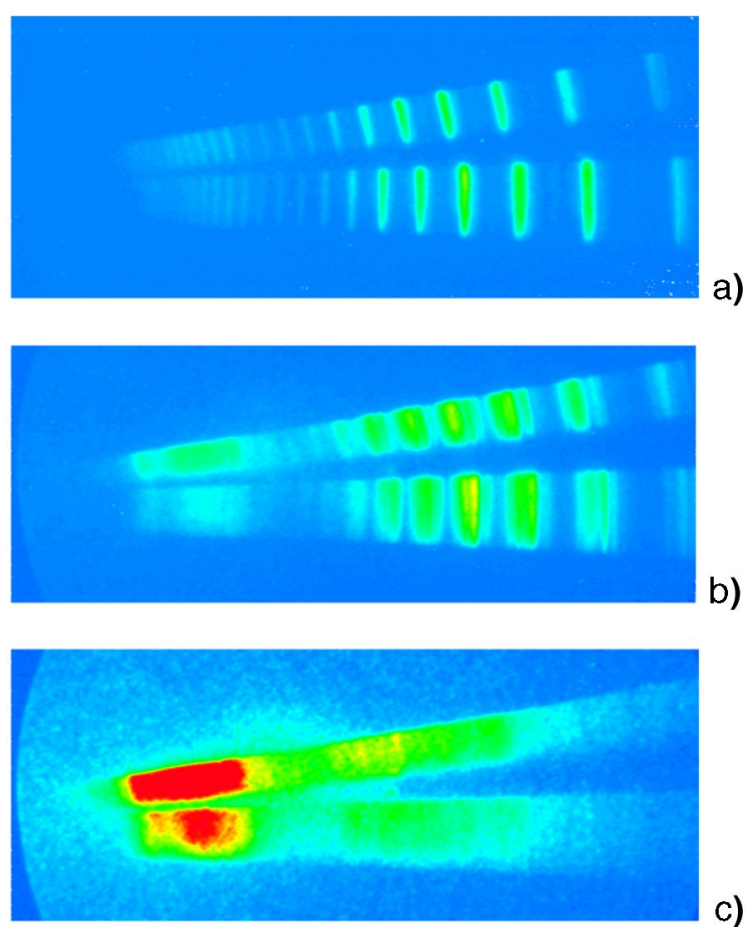

FIG. 2 (color online). Raw data from experiments 7th (right) to 25th (left) harmonic ( $s$ polarization on top, $p$ polarization below). (a) Harmonics from front of target, (b) harmonics from rear of target $(0.2 \mu \mathrm{m} \mathrm{Al})$, (c) harmonics from rear of target $(0.4 \mu \mathrm{m} \mathrm{Al})$. Note that the color scale has been changed between (a), (b), and (c) in order to show the spectra clearly. The absolute level of the short wavelength signal in (b) is similar to that in (c).

the rear surface of thin targets can be a source of harmonic radiation up to the 10th order due to the coupling of the plasma to the temporally modulated fast electron beam in the plasma [16]. However, these experiments are clearly in a different regime than in our experiment where the intensity of the interaction was almost 2 orders of magnitude larger and the prepulse intensity was consequently much higher. This implied that the density profile was also quite different in these situations. In addition, the spectral line shapes of the individual polarization components in our experiment are different for each harmonic indicating that these different line shapes are due to interaction of the radiation with the plasma after generation rather than due to the harmonic production mechanism.

It is also clear from our measurements that the dense plasma of the target was opaque to the lower-order harmonics. Indeed a shift towards transmission of higherorder harmonics was observed when the initial thickness of the foil and corresponding density of preheated plasma was increased (Fig. 2). Only radiation with high enough frequencies for which the target was underdense could be observed in the rear side. Accordingly in our theoretical model the high frequency harmonics originate at the critical surface and propagate through relativistic plasma produced from thin foil targets. The index of refraction of this 
plasma, $\eta=k c / \omega$, is derived from the kinetic equation assuming two electron populations: cold background (with $n_{c}$ as the density and $T_{c}$ the temperature) and a hot one having relativistic energies $\left(n_{h}, T_{h}\right)$. This has been confirmed by previous experiments and simulations in this regime $[1,2,11]$. The hot electron distribution function is a relativistic Maxwellian, $f_{h} \sim \exp \left[-c\left(m^{2} c^{2}+\right.\right.$ $\left.p^{2}\right)^{1 / 2} / T_{h}$. In very thin foil plasmas, fast electrons, which drive target backside expansion, are reflected and recirculated in the target producing a symmetric momentum distribution function [17]. The hot electron temperature $T_{h}$ is then estimated from the ponderomotive [18] scalings $\left(T_{h} \sim I_{0}^{1 / 2}\right)$ in the range of $4 \geq T_{h} \geq 2 \mathrm{MeV}$. The temperature of background electrons is more difficult to estimate. We have taken $T_{c} \ll T_{h}$ with $1 \leq T_{c} \leq 10 \mathrm{keV}$ in these targets assuming that cold electrons are Maxwellian. From the electromagnetic wave $(\omega, k)$ dispersion relation in the limit of $m c^{2} / T_{h} \ll 1, k c / \omega<1$, one obtains [19]

$$
\left(\frac{k c}{\omega}\right)^{2}=1-\left(\frac{\omega_{p c}}{\omega}\right)^{2}-\frac{1}{3} \frac{m c^{2}}{T_{h}}\left(\frac{\omega_{p h}}{\omega}\right)^{2},
$$

where $\omega_{p i}=\left(4 \pi e^{2} n_{i} / m\right)^{1 / 2}(i=c, h)$. A frequency shift and spectral broadening of the transmitted harmonic radiation are caused by the time variation of the index of refraction (1) along the wave's optical path. The index of refraction (1) changes in time because of the varying relative concentration of cold and hot electrons and target expansion. Because of the absorption of a large fraction of the pulse energy, the majority of the cold electrons in the interaction region will be heated to relativistic energies. However, in spite of increasing $n_{h}$ contributions of fast electrons to the index of refraction (1) are small because of the factor $m c^{2} / 3 T_{h} \sim 0.04$ for $T_{h}=4 \mathrm{MeV}$. Indeed the main effect on the index of refraction (1) is due to the "loss" of cold electrons. Note that ionization effects would give rise to a frequency blueshifted "tail" of the spectrum rather than the observed redshift of the transmitted harmonics.

To describe the evolution of a target we have solved a one-dimensional model [20] including the ion Vlasov equation, the Poisson equation for the plasma potential $\phi(x, t)$, and Boltzmann distributions for the electron density, $n_{e}=n_{c} \exp \left(e \phi / T_{c}\right)+n_{h} \exp \left(e \phi / T_{h}\right)$. Temperatures $T_{c}, T_{h}$ are kept constant because of the short time scales of the interaction (note that this approximation does not affect results significantly). We have introduced a temporal variation of $n_{h}(t)$ and $n_{c}(t)$ to model laser light absorption. We have used the energy balance, $3 T_{h} N_{h} / 2=\int_{0}^{t} d t^{\prime} I_{\text {abs }}\left(t^{\prime}\right)$ between fast electrons and the absorbed laser flux $I_{\text {abs }}=$ $A I(t)$, where $A$ is the averaged absorption coefficient and $N_{h}(t)=\int_{0}^{\infty} d x n_{h}(x, t)$ is the total number of fast electrons. Also the particle number conservation, $n_{e}(t=0) L_{0}=$ $N_{c}(t)+N_{h}(t)$, has been employed to define time variations of the cold electron number, $N_{c}(t)=\int_{0}^{\infty} d x n_{c}(x, t)$, where $L_{0}$ is the initial width of the plasma $\left(5<L_{0}<10 \mu \mathrm{m}\right)$. In the numerical implementation of our model we have used a

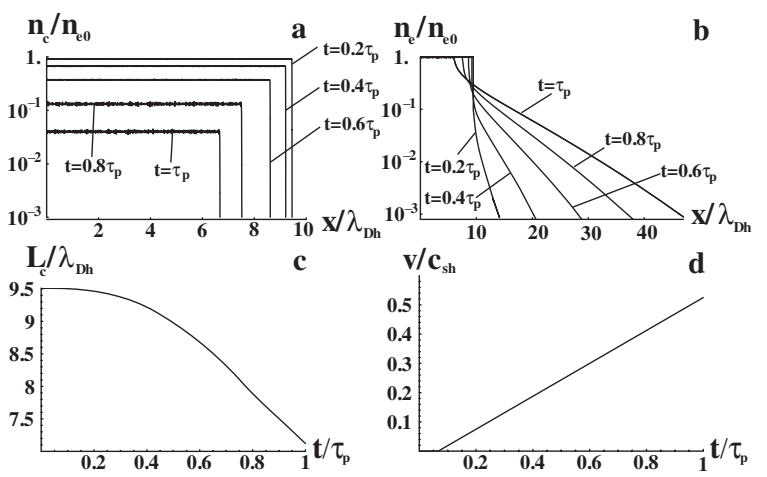

FIG. 3. Profiles of the cold electron density (a) at different moments in time and the total electron density (b). Time evolution of $L_{c}$, which marks the sharp shocklike interface between cold electrons and the rest of the plasma (c). Velocity of the $L_{c}$ interface $(\mathrm{d})$.

simplified form of the laser pulse $I(t)=I_{0} \sin \left(\pi t / \tau_{p}\right)\left(\tau_{p}\right.$ is the pulse duration) and have introduced two additional approximations: $N_{h} \sim n_{h}(t) L_{0}$ in the energy balance condition and $N_{c} \sim n_{c}(t) L_{c}(t)$ in the particle conservation law. The above form of $N_{c}$ is based on simulation results where $L_{c}(t)$ is the position of the rarefaction shock which confines decreasing number of cold electrons to the region of the plasma of $\phi \sim$ const and $n_{c} \sim \delta n_{c}$. Backside expansion of the target is illustrated in Fig. 3. This is a result of the Vlasov simulation for $T_{h}=4 \mathrm{MeV}, T_{c}=1 \mathrm{keV}, n_{e}(t=$ $0) \approx n_{c}(t=0)=5 \times 10^{22} / \mathrm{cm}^{3}, L_{0}=10 \mu \mathrm{m}$, and $I_{0}=$ $10^{20} \mathrm{~W} / \mathrm{cm}^{2}$ and $\tau_{p}=800 \mathrm{fs}$. At the beginning of the pulse the number of fast electrons is small $\left(n_{h}<0.01 n_{c}\right)$; however, due to large absorption their number is comparable with the total number of electrons at the end of the pulse. Figure 3(a) shows the cold electron density $n_{c}$ at several moments in time. The existence of this rarefaction shock has been predicted in Ref. [20] for a two-electron temperature plasma and $T_{h} / T_{c} \geq 9.9$. For $T_{h} / T_{c}=4000$ in our simulations the shock can already form at $n_{h} / n_{c}<$ 0.1 according to Ref. [20]. Plasma expansion driven by the fast electron population is shown in Fig. 3(b). The dynamics of this process depends on the time varying laser intensity and the varying plasma composition which results in almost constant acceleration of the cold electron interface and a reduction in the value of $L_{c}(t)$ [Figs. 3(c) and 3(d)]. Previous work [21] indicates that a 1D approximation for the rear surface expansion is reasonable for interpreting the results of such experiments.

The oscillating plasma mirror model $[9,10,22]$ has been introduced to explain higher harmonic generation in short pulse laser dense plasma interactions through the nonlinear dynamics of the critical surface using the cold electron fluid model approximation. The oscillating surface modulates the waveform of the incident sine wave which consequently contains higher-order harmonics during the interaction. For frequencies larger than $5 \omega_{0}$ they can also propagate through the overdense plasma of the preheated foil and contribute to the spectra observed at the back side 


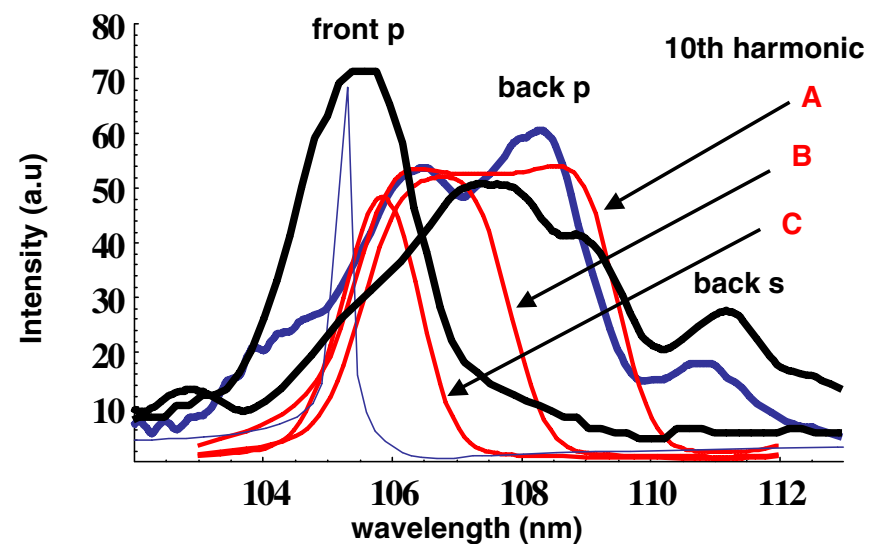

FIG. 4 (color online). Different 10th harmonic spectra compared with the oscillating mirror model. Comparison of experimental harmonic profiles with different frequency shift calculations for three values of the hot electron fraction (A) $n_{\text {hot }}=0.95 n_{e},(B) n_{\text {hot }}=0.53 n_{e},(C) n_{\text {hot }}=0.21 n_{e}$. The best fit for the experimental data occurs with $(A)$. Note that the initially uncorrected oscillating mirror model spectrum has been adjusted to account for the spectral resolution of the spectrometer in $(A),(B)$, and $(C)$.

of the target. Following [10] we describe the critical surface motion in terms of the superposition of oscillations at two frequencies $\omega_{0}$ and $2 \omega_{0}$. This leads to the oscillating mirror model spectrum in Fig. 4. Positions of the calculated harmonics are in good agreement with the observed spectrum at the front of the target. Harmonics observed in the backside display redshift, broadening, and additional structure in the spectrum due to plasma effects as observed in the experiment.

Higher harmonics, which propagate through the target, can therefore provide an optical diagnostic of dense relativistic plasmas. Their spectral properties, such as the observed redshift, are due to time variations of the index of refraction (1) occurring due to plasma evolution as shown in Fig. 3. We use the spectrometer instrumental resolution $(<1 \mathrm{~nm})$ in order to compare measurement with the oscillating mirror model calculations. The frequency shift of the $p$-polarized 10th order harmonic behind the target is shown in Fig. 4. We have calculated its magnitude by taking the Fourier transform of the plane wave $\exp \left[-i \omega_{10} t+i \delta \varphi_{10}(t)\right]$ where the phase factor is $\delta \varphi_{10} \sim\left(\omega_{10} / c\right) \int d z^{\prime} \eta_{10} \sim-\omega_{10} n_{c}(t) L_{c}(t) /\left(2 c n_{\mathrm{cr}}^{10}\right)$. The symbol $n_{\mathrm{cr}}^{m}$ stands for the critical density of the $m$ th harmonic, $\omega_{10}=10 \omega_{0}$, and we have used the approximation $n_{c} / n_{\mathrm{cr}}^{10} \ll 1$ in calculation of $\delta \phi_{10}$. Variations of the cold electron density and $L_{c}(t)$ are given by the simulation results. In Fig. 4 we have shown three cases of the frequency shifts which are calculated for different hot electron fractions. Only for higher hot electron density $(95 \%$ of background density) is the redshift of the 10th harmonic comparable with experimental observations. Smaller values of $n_{\text {hot }}$ result in smaller fractions of cold electron population heated to relativistic energies during the interaction. Also in these simulations we have increased the initial value of $L_{c}$ by 2 to account for the longer optical path for harmonics which are observed off the target normal direction. Note that magnetic fields in the interior of the target due to the hot electron current may also induce ellipticity in the polarization of light through the CottonMouton effect [23]. This was observed previously in the harmonic spectrum on the front side of the targets [24] and may also be the cause of the observed $s$ component in the transmitted harmonics.

In conclusion, the observed spectral features of the harmonic radiation measured from the rear of thin foil targets are observed to agree with theory and modeling, thus giving the first direct evidence of the existence of very high density $\left(n_{\text {hot }}>10^{21} \mathrm{~cm}^{-3}\right)$ relativistic plasma in the laboratory.

The authors would like to acknowledge support from Russian Foundation of Basic Research (RFBR) Grant No. 06-02-16103, NSERC of Canada, and the UK EPSRC. M.Z. would also like to thank the Royal Society Wolfson Foundation and the Office for Science and Technology for additional support.

*Present address: Center for Ultrafast Optical Science, University of Michigan, Ann Arbor, MI 48109, USA.

[1] M. I. K. Santala, et al., Phys. Rev. Lett. 84, 1459 (2000).

[2] M. H. Key et al., Phys. Plasmas, 5,1966 (1998).

[3] E. L. Clark et al., Phys. Rev. Lett. 84, 670 (2000); R. A. Snavely et al., Phys. Rev. Lett. 85, 2945 (2000).

[4] K. W. D. Ledingham et al., Phys. Rev. Lett. 84, 899 (2000).

[5] M. Tatarakis et al., Nature (London) 415, 280 (2002).

[6] K. Krushelnick et al., Plasma Phys. Controlled Fusion 44, B233 (2002).

[7] B. Dromey et al., Nature Phys. 2, 456 (2006).

[8] P. A. Norreys et al., Phys. Rev. Lett. 76, 1832 (1996).

[9] S. V. Bulanov, N. M. Naumova, and F. Pegoraro, Phys. Plasmas 1, 745 (1994).

[10] R. Lichters, J. Meyer-ter-Vehn, and A. Pukhov, Phys. Plasmas 3, 3425 (1996).

[11] R. B. Campbell et al., Phys. Rev. Lett. 94, 055001 (2005); S. P. Hatchett et al., Phys. Plasmas 7, 2076 (2000); J. Myatt et al., Phys. Plasmas 14, 056301 (2007).

[12] M. Tabak et al., Phys. Plasmas 1, 1626 (1994).

[13] C. N. Danson et al., J. Mod. Opt. 45, 1653 (1998).

[14] G. J. Pert, J. Comput. Phys. 43, 111 (1981).

[15] R. Hassner et al., Opt. Lett. 22, 1491 (1997).

[16] U. Teubner et al., Phys. Rev. Lett. 92, 185001 (2004); H. Popescu et al., Phys. Plasmas 12, 063106 (2005).

[17] A. J. Mackinnon et al., Phys. Rev. Lett. 88, 215006 (2002).

[18] S. C. Wilks et al., Phys. Rev. Lett. 69, 1383 (1992).

[19] V. P. Silin, Sov. Phys. JETP 11, 1136 (1960).

[20] V. Yu. Bychenkov et al., Phys. Plasmas 11, 3242 (2004).

[21] S.C. Wilks et al., Phys. Plasmas 8, 542 (2001); A. A. Andreev et al., Laser Part. Beams 22, 431 (2004).

[22] B. Bezzerides, D. Forslund, and E. Lindman, Phys. Fluids 21, 2179 (1978); V. T. Tikhonchuk et al., Plasma Phys. Controlled Fusion 47, B869 (2005).

[23] M. Tatarakis et al., Phys. Plasmas 9, 3642 (2002).

[24] U. Wagner et al., Phys. Rev. E 70, 026401 (2004). 